\title{
UNA IDEA, DOBLE RECEPCIÓN: LAS TRES JUSTICIAS EN UNA Y LA MOJIGATA
}

\author{
ONE IDEA, DOUBLE RECEPTION: \\ LAS TRES JUSTICIAS EN UNA AND LA MOJIGATA
}

\author{
ISMAel López Martín \\ Universidad de Extremadura. Cáceres, España \\ ismael@unex.es
}

\begin{abstract}
Resumen: Este artículo analiza dos textos que pertenecen a la dramaturgia clásica española: Las tres justicias en una de Pedro Calderón de la Barca, y La mojigata de Leandro Fernández de Moratín. Estudiamos los componentes de sus acciones, sus personajes, sus recursos estilísticos, sus temas y sus significados. Teniendo en cuenta que pertenecen a diferentes siglos (XVII y XVIII) y géneros (comedia nueva y comedia neoclásica), al final del trabajo comparamos las dos obras desde todos sus puntos de vista (incluyendo sus relaciones con algunos tratados teóricos sobre teatro de cada época) para observar el distinto tratamiento que hacía el teatro de uno de sus temas más destacados, la educación de los hijos, que aparece en ambos textos.
\end{abstract}

Palabras clave: Teatro clásico español, Calderón, Moratín, educación de los hijos.

\begin{abstract}
This article analyzes two texts belonging to Spanish classical dramaturgy : Las tres justicias en una, by Pedro Calderón de la Barca, and La mojigata, by Leandro Fernández de Moratín. We study their actions, characters, stylistics resources, themes and meanings. Considering they belong to different centuries (XVII and XVIII) and genres (new comedy and neoclassical comedy), at the end of the paper we compare the two works from many different points of view (including their relationship with some theoretical treatises on drama of each time period) to observe the different treatment that they make of a salient theme, the education of their children, which appears in both texts.
\end{abstract}

Palabras claves: Spanish Classical theatre, Calderón, Moratín, children's education.

Recibido: 10.08.2014. Aceptado: 10.04.2015. 


\section{Las tres justicias en una}

$\mathrm{E}$ stamos ante una de las comedias de don Pedro Calderón de la Barca (1923) en las que no hay un consenso en torno a su datación ni antecedentes del argumento. Por un lado, Hilborn (1938, pp. 36-40) cree que la fecha de composición de la obra es 1636, aunque no conservamos ni el manuscrito ni ninguna fuente indirecta que nos dé la fecha exacta. Más prudente es el análisis métrico de Parker (1962: 229), que la sitúa en una horquilla que va de 1635 a 1640. En cualquier caso, sabemos que Pedro de Ascanio la intentó representar en Valencia en 1644 y que la primera vez que apareció impresa fue en 1661, en la Parte quinze de las Comedias nuevas, escogidas de los mejores ingenios de España.

De su estudio se extraen dos fuentes básicas (Benabu, 1991): la Segunda Parte de El príncipe perfecto (1623) de Lope de Vega y la Chronica dos reys de Portugal (siglo XIV), en las que se pone de manifiesto la falta de respeto de un hijo a su padre. Seguramente Calderón situó la obra en Aragón porque era más cercano que Portugal para el público barroco.

Calderón revela una compleja red de relaciones familiares que sirven para desarrollar los temas más importantes y de más aceptación entre el público barroco: el amor, la religión, el honor y la familia. La función de enlace que don Lope de Urrea hijo tiene entre las dos acciones que componen el argumento (relación familiar y relación amorosa de don Lope) viene a determinar una clara separación por actos a la hora de contar las historias: el primer acto resume las relaciones familiares, el segundo se encarga de las amorosas y el tercero relaciona ambas.

En el acto I encontramos esa introducción al espectador de las redes familiares a través de incidentes anticlimáticos y recursos dramáticos como la retrospección y los soliloquios. Así, se presenta al auditorio la historia de don Lope de Urrea hijo desde tres vertientes. En primer lugar, es el propio don Lope hijo quien hace un repaso a su historia personal (vv. 137-162 y vv. 166-372) desde el punto de vista de su padre, de quien pretende distanciarse enjuiciándolo y justificando sus malos actos de cara a una futura resolución final del conflicto. En segundo lugar, será don Lope padre el que se dirija al Rey (vv. 451-541) pidiendo justicia para su hijo retomando la historia que este le estaba contando a su interlocutor; en esta ocasión el padre se centra en la figura de su hijo, censurando su comportamiento, 
por lo que los asistentes a la representación tendrían una doble visión del conflicto: venturas y desventuras del padre y del hijo relatadas por ellos mismos. Por último, será doña Violante la que, brevemente, recuerde, al final del acto, el inicio de la obra, para, de ese modo, comenzar el acto II con toda la información actualizada.

Calderón emplea unas situaciones anticlimáticas en este primer acto, predominando, como es lógico, las escenas narradas a las escenificadas. Los tres incidentes importantes de esa primera jornada, que son la base para el desarrollo de los temas, son la boda con una gran diferencia de edad entre don Lope de Urrea padre y doña Blanca, el incumplimiento de la palabra de esposo y la salvación que don Lope hijo hizo a doña Violante.

$\mathrm{El}$ acto II se centra en las relaciones amorosas de don Lope de Urrea hijo quien, como galán, corteja a doña Violante dentro de los cánones de un amor cortés evolucionado. Hay, además, un triángulo amoroso complejo en el que la dama se sitúa en el vértice superior, aunque la verdadera complejidad radica en la base, conformada por don Lope de Urrea hijo y don Guillén de Azagra, que utiliza al primero como tercero para acercarse a doña Violante sin saber que su competidor también la pretende, conformándose un entramado cómico que tiene su culminación en el paralelismo que los criados conforman, ya en un nivel satírico-burlesco.

El acto III no es sino una constante exposición de doctrina moral sobre el respeto que los hijos deben a sus padres, y por ello aparecerá amarrado don Lope hijo en escena, censurando el autor todo este tipo de comportamientos. Nuevamente la obra vuelve a caer en algunos recursos del primer acto, como son los monólogos que cuentan la prehistoria de la comedia, esta vez para informar al Rey y plantear al público una justificación de las justicias que se tomarán al final del texto para solucionar todos los problemas.

En tanto que obra literaria, está compuesta de tal modo que la genialidad del dramaturgo se observa en la perfecta simbiosis de todas las partes que conforman una obra: acción, personajes, temas, significado y recursos dramáticos, verdaderas herramientas de las que se sirve el escritor para lograr en el espectador y en la escena todas esas situaciones y predisposiciones de las que se sirve el hecho teatral. Aparece un comienzo in medias res, pues Calderón no empieza esta historia por el principio, sino que, seguramente para captar de un modo más rápido la atención del espectador, 
abre el telón con un hecho climático y de gran espectacularidad: una persecución. Aparecen también alusiones mitológicas para caracterizar tanto física como psicológicamente a los personajes, atribuyéndoles ciertas dotes o propiedades que la tradición grecolatina distinguió entre algunos de sus dioses o héroes. Juega un papel fundamental el contraste, siendo el principal conflicto de la obra la mala relación entre don Lope de Urrea y su hijo. Por eso este recurso no solamente se va a centrar en oponer incidentes, sino que distanciará los caracteres y los propios personajes de don Lope y su hijo y los sentimientos que generan dentro de su propia familia.

La oposición entre apariencia y realidad también está presente en la comedia, y la herramienta del disfraz puede aplicarse al tema de las relaciones paterno-filiales, pues es el medio que tiene el personaje para no descubrirse ante su padre y desatar, así, nuevas disputas. Determinados elementos físicos u objetos se identifican con valores morales, jurídicos, políticos, con personajes o simplemente con incidentes principales o secundarios dentro de una de las acciones: es el simbolismo. Por su parte, la anagnórisis es un recurso derivado del comienzo in medias res y de la oposición entre apariencia y realidad, pues el público ha de tener toda la información; en ocasiones la verdad es revelada únicamente a los personajes de la obra, sabiéndolo ya el auditorio, como cuando doña Blanca dice a doña Violante que aquel por quien suspira es su hijo (v. 751).

Otros recursos que aparecen en la pieza son las alusiones religiosas e históricas, el paralelismo, el lirismo, el triángulo amoroso, el enredo y la expectación, las oposiciones binarias, la perspectiva múltiple o la justicia poética.

En cuanto a los personajes, Beatriz y Elvira están construidas sobre el tipo del "criado", y no hay mayores diferencias entre ellos: son consejeras de su ama, transmisores de noticias y, en definitiva, unos personajes de relación entre incidentes secundarios.

Vicente está construido sobre los tipos del "criado" y del "gracioso": acompaña a su amo, aconsejándole, y participa en el desarrollo de un triángulo "amistoso" y burlesco, porque en realidad Vicente no quiere servir a una de las otras dos criadas, sino que se dedica a injuriarlas hipócritamente mediante descripciones físicas que no se ajustan a la realidad.

Don Mendo Torrellas es un anciano dotado de experiencia y venerabilidad. Está, de este modo, basado en el tipo del "viejo"; pero, sin duda, 
también está construido sobre el "poderoso" que ha ascendido social y políticamente con trabajo y dedicación, y lealtad al Rey, quien es un "poderoso" total, pues por la autoridad que le ha sido conferida imparte justicia al final de la comedia. Probablemente el público podría pensar que esta justicia no es justa, pues escenas atrás se demostró que la relación paterno-filial entre los dos don Lope no existía, invalidando así el delito de irrespetar al padre. Sobre la identidad histórica del rey, tenemos claro que se trata de un rey llamado don Pedro y al que se conocía con los sobrenombres de "Cruel" y "Justiciero" en función de que los que así lo llamaban fueran sus detractores o sus partidarios, respectivamente. La ausencia en el texto del numeral ordinal que acompaña a los nombres de los reyes dificulta considerablemente su identificación, pues esos apelativos pueden corresponder a tres monarcas contemporáneos entre sí: Pedro I de Portugal (1357-1367), Pedro IV de Aragón (1336-1387) y Pedro I de Castilla (1350-1369). Pedro I de Portugal cabría porque el argumento podría estar basado en un suceso real y documentado que ocurrió durante el reinado de ese monarca en Portugal. Pedro IV de Aragón se corresponde con el lugar de donde don Pedro era rey en la comedia, pero el apelativo que se le adjudicó fue el de "Ceremonioso". Sin embargo, en territorio hispano sí que se tuvo especial conocimiento de que a Pedro I de Castilla lo llamaban "Cruel" o "Justiciero". La comedia nos ofrece algunos datos históricos: nos habla de la Guerra de Nápoles (1284-1285) y nos dice que de eso han pasado tres años, por lo que situamos la obra en 1287. Era rey de Aragón don Alfonso III, sucesor de Pedro III el Grande desde 1285. Calderón leería la Chronica dos reys de Portugal y seleccionaría el pasaje mencionado, situándolo en la Corona de Aragón por ser un lugar más próximo a los españoles del siglo XVII que Portugal, mantuvo los sobrenombres del rey portugués e incluyó el suceso histórico de la Guerra de Nápoles en la obra porque lo creyó oportuno (no tiene una importancia extrema en el texto), aun teniendo que someterse a un anacronismo que poco importaba a los espectadores, ya lejanos de esa última batalla que los aragoneses ganaron hasta la época de, ya sí, Pedro IV. En resumen, con una acción situada en Aragón, el personaje del rey don Pedro se corresponde con Pedro I de Portugal, "el Cruel" o "el Justiciero".

Don Guillén de Azagra es un personaje claramente construido sobre el tipo del "galán". Se trata de un joven del que no se da ninguna descripción física, aunque sí conocemos algunos rasgos de su carácter: es confiado, 
impetuoso, valiente e inconsciente. Desarrolla perfectamente el tema del amor y genera incidentes climáticos.

Doña Blanca es un personaje formado desde el tipo de la "dama" y que cumple a la perfección las facultades atribuidas a ese tipo: se trata de una joven bella que está casada con un hombre mayor (desarrollando el tema de los matrimonios entre edades desiguales: el tópico del viejo y la joven), es discreta, pasiva y sus intervenciones en la comedia se limitan a los largos parlamentos que le sirven para recordar su infancia e historia personal y para relatar al Rey los incidentes sobre los que ha de juzgar.

Doña Violante es la hija de don Mendo Torrellas y se basa, también, en el tipo de la "dama". Desarrolla el tema del amor y es protagonista del triángulo amoroso que forma con don Lope hijo y don Guillén.

Don Lope de Urrea padre es "viejo", aunque también participa en el tema del amor a través de la vertiente de los matrimonios desiguales con respecto a la edad. Pero es el tema del honor el que tiene un mayor desarrollo con este personaje. Se permite aconsejar a su hijo y dirigirlo hacia los buenos modales y comportamientos morales.

Don Lope de Urrea hijo es un "galán", y es el personaje principal de toda la comedia, por lo que su caracterización es la más compleja. Pero, más que por los rasgos que presenta, de los que físicamente Calderón no aporta ninguno, por los temas que introduce. Es especialmente importante para desarrollar los tres temas que se dan en la obra: relaciones paterno-filiales, honor y amor, convirtiéndose en el eje compositivo de los tres.

Los temas que trata la obra tienen puntos de confluencia. Así, Las tres justicias en una comienza con el de las relaciones paterno-filiales. Don Lope padre recuerda que estos sucesos perjudican la honra familiar, enlazando ahora con ese tema de la honra. Como don Lope hijo ya estaba libre de compromisos, puede dedicarse a la galantería, al tema del amor, que debido a su escasa importancia en la obra ni siquiera se soluciona, aunque todo su desarrollo se sitúa en la parte central de la comedia. Ofrecemos a continuación una pequeña sucesión esquemática de los temas para una mayor comprensión de su importancia y su interrelación en el conjunto de la comedia:

Relaciones paterno-filiales / Honra / Amor / Honra / Relaciones paterno-filiales. 
Se observa una disposición simétrica en la que la importancia de los temas aumenta en función de la distancia al centro.

A lo largo de la obra no se intuye casi ninguna sentencia didáctica, únicamente se enjuicia el comportamiento de don Lope hijo, pero él no es una mala persona, es un inconformista con las leyes naturales y morales de la época y quiere ser libre para elegir su propio destino. Calderón plasma sus convicciones personales en la obra, condenando el comportamiento de don Lope hijo con todas sus fuerzas y un estilo ejemplar para recordar a los espectadores que no se puede faltar a la palabra de esposo y que no se puede irrespetar a un padre.

\section{La Mojigata}

Fue escrita a finales del siglo XVIII y se incluye en el género de la comedia neoclásica o comedia de buenas costumbres. Circularon copias escritas desde 1791, pero Moratín (1825, pp. 3-4) no pudo representarla hasta 1804. No fue un dramaturgo que cosechó muchos éxitos con sus obras, aunque con La mojigata pudo disfrutarlo, pero no en exceso.

Aunque pudo basarse en textos anteriores como El lindo don Diego de Agustín Moreto o Las tres justicias en una de Calderón (que ya se ha analizado), en los que aparece el tema de la educación de los hijos y de la libertad de elección de pareja, seguramente tomó numerosos elementos de La petimetra de su padre, Nicolás Fernández de Moratín, que tanto criticó.

En La mojigata (Fernández de Moratín, 1977), y en clara contraposición con Las tres justicias en una, se observa una trama amorosa en la que prima la obediencia o no a los padres, y con base en ello se construirá la acción mediante una serie de incidentes principales y secundarios, climáticos y anticlimáticos. Los incidentes principales se referirán siempre a la acción amorosa, mientras que los secundarios pueden vincularse a esas relaciones o a momentos puntuales en los que el autor quiere desviar la atención de los espectadores para no cansarlos mediante la inclusión de sucesos paralelos y que poco tienen que ver con la historia principal.

En el acto I nos encontramos un planteamiento del conflicto amoroso de la comedia, pero a la vez de las malas relaciones paterno-filiales entre 
don Martín y doña Clara. Es doña Clara el personaje de enlace entre ambos temas, aunque, como comprobaremos más adelante, el de la familia es considerablemente más importante que el del amor, y esto se debe también a que la aparición de incidentes principales y climáticos es menor en este tema. En esta primera parte, don Martín y don Luis, su hermano, debaten sobre la educación de los hijos y el papel que tienen los padres, aunque no hacen digresiones demasiado teóricas, sino que se centran en las figuras de sus hijas.

En el acto II se urde una trama de relaciones de conveniencia derivadas de los amores entre don Claudio y doña Clara. Así, Lucía es requerida tanto para ayudar a don Luis como a don Claudio, convirtiéndose en el eje de esta nueva situación y desarrollando aún más esa función característica del tipo del criado que es ser confidente. El único impedimento que la relación amorosa encuentra para poder llevar a buen término es la oposición de don Martín, y por eso los personajes principales y sus criados tendrán especial cuidado en no desvelarle nada, por lo que la expectación tendrá mucha importancia en este acto. Don Luis y don Martín tendrán un diálogo en cada acto que tiene una gran carga moral sobre el tema principal de la comedia: la educación de las hijas en la contemporaneidad de la obra.

El acto III supone la anagnórisis de don Martín sobre la relación y futuro matrimonio entre don Claudio y su hija y la resolución de los conflictos. Al final, y como resumen moral que no podía faltar en una obra neoclásica, la hija es perdonada, aceptando el padre el destino de los tiempos de renovación que se estaban viviendo, entre otros, en la autoridad paterna y en la capacidad de decisión de los hijos sobre su matrimonio.

También en La mojigata aparece la introducción in medias res: Moratín propone una historia ya comenzada con el fin de captar la atención del público desde el primer momento e introducirlo en el tema de la educación de los hijos. Se incluyen retrospecciones, es decir, el recuerdo de sucesos pasados o incluso no representados porque se sitúan en la prehistoria de la obra o de los personajes. Por su parte, la prospección se encarga de adelantar acontecimientos, por lo que afecta especialmente a la acción. El autor mantiene la atención del público al presentar como futuribles determinados sucesos que ellos esperan. La introspección muestra los sentimientos más profundos de los agonistas, relacionándose con el recurso del lirismo 
debido a la altura de la expresión. Se trata de un recurso utilizado por los comediógrafos para conmover al público en torno al tema del amor.

En determinadas ocasiones, los personajes se ven obligados a hacer un guiño al espectador y dirigirle unas palabras sin que, se supone, las escuche ningún otro personaje, son los apartes. De este modo, se crea un vínculo de complicidad entre el dramaturgo y el público, pues se trata de pequeños detalles que, además, mantienen despierto el interés del auditorio y le hacen sentirse valorado por el creador. Estos apartes son especialmente importantes para el desarrollo de la acción, y en cierta medida tienen mucho que ver con las oposiciones entre apariencia y realidad, pues un personaje comunica en voz muy baja determinadas situaciones básicas para crear incidentes climáticos y generar tensión, lo cual no se lleva a cabo precisamente por ese sigilo.

La estética neoclásica propugnaba unas obras de teatro construidas específicamente en función de una enseñanza moral aplicable a la vida cotidiana de los espectadores. En numerosas ocasiones se trata de sentencias breves entresacadas del comportamiento de ciertos personajes a modo de síntesis, y hemos de indicar que en La mojigata se trata de unas sentencias ex contrario, es decir, se explica el comportamiento negativo y se reprueba mediante una máxima.

La alusión literaria es un recurso que muestra citas o referencias a determinadas obras, temas, autores o ideas de la historia de la literatura universal. La funcionalidad de este recurso reside en la ejemplificación de determinados comportamientos o situaciones, la ilustración del público (que no encontramos en nuestra obra) o la simple mención. Así, en el verso 866, cuando se cita a un "convidado de piedra", puede hacer referencia a la obra de Tirso de Molina titulada El burlador de Sevilla y convidado de piedra (de 1630) o a No hay plazo que no se cumpla ni deuda que no se pague, y Convidado de piedra, de Antonio de Zamora. En el verso 1450 se cita a Marcolfa y Cacaseno, y esto lleva a una colección de tres cuentos populares, Bertoldo, Bertoldino y Cacaseno, escritos por Croce y Banchieri y publicados en 1620 .

También están presentes el contraste, el enredo, la anagnórisis, el triángulo amoroso, la expectación, las alusiones históricas, los paralelismos, los enfrentamientos duales, el simbolismo y los resúmenes didácticos. 
El número de personajes que aparece en La mojigata es de ocho, con unas funciones muy diferenciadas y arquetípicas, si bien es cierto que, excepto en el caso del galán y del Tío Juan, podríamos establecer dualidades de personajes: los padres, las damas y los criados.

El Tío Juan no está construido sobre ningún tipo dramático, por lo que sus funciones únicamente se desprenden de sus dos breves intervenciones en escena y que constituyen unos incidentes anticlimáticos, pues se alejan de la acción principal.

Don Claudio es un "galán" aunque, como en el caso anterior, tampoco encontramos una especial caracterización del personaje; cumple las funciones que tradicionalmente se le atribuyen: forma enredo, ama a la dama y mantiene diálogos sobre el amor con ella y su criado, a quien, en ocasiones, utiliza como confidente. Tiene ciertas características de "dama", pues no posee una autoridad destacable, sino que es sumiso a las decisiones de la dama y del padre. Aunque manifiesta su opinión y su amor por doña Clara, no lucha tenazmente por ella y contra su padre, como sí hará la dama, sino que se dedica a aceptar lo que el destino le depare. Hemos de decir que don Claudio también está construido sobre el "figurón", al menos en algunos rasgos, y de ahí su interés por casarse con doña Clara y obtener la sustanciosa herencia que don Martín tiene para su hija.

Perico y Lucía son los criados, y ambos están construidos sobre este tipo. Como criados son confidentes, terceros, transmisores de noticias... Sus amos, don Claudio y doña Clara, hablan con ellos de sus cuitas amorosas, recurriendo frecuentemente al recurso de la prospección cuando le confiesan sus planes futuros de encuentros amorosos. Además, y como venía siendo tradicional desde la comedia barroca, los criados conforman un paralelismo amoroso con respecto a sus amos, aunque su relación está bastante más perfilada desde el principio que la de los señores. Pero la caracterización de Perico y Lucía no es uniforme, no en vano el criado está construido también sobre el tipo del "gracioso" y la criada no. Perico es considerablemente más completo que Lucía, quien únicamente participa de sus funciones de criada y confidente de los amores de su señora. Perico, en cambio, como "gracioso" protagoniza la única escena en la que aparece el Tío Juan, introduciendo en ese y otros puntos de la obra momentos anticlimáticos.

Don Martín y don Luis son los padres de las damas. El tipo del "poderoso" sirve a Moratín para perfilar ambas personalidades y como tal ejercen 
la autoridad (en este caso, familiar) que poseen, afectando a los tres temas fundamentales de la obra: el amor, las relaciones paterno-filiales y la educación de los hijos. En ambos casos, y cada uno a su manera, pretenden el mayor bien posible y la felicidad para sus hijas, pero los medios para llevarla a cabo son algo distintos.

Doña Clara y doña Inés son primas y su comportamiento es radicalmente opuesto. Es cierto que ambas están construidas sobre el tipo de la "dama", y por ello son destinatarias (realmente solo doña Clara) del amor de un galán. Aparte de la belleza, no hay ninguna otra caracterización de estos dos personajes, por lo que debemos extraer la información de sus intervenciones en la pieza. Si analizamos sus funciones en la comedia nos percatamos de que doña Clara también se construye sobre el tipo del "galán” y tiene algunas características que le faltan a su amado: la valentía y el arrojo suficientes para enfrentarse a don Martín como medio para conseguir la ansiada libertad. Mientras que doña Inés es sumisa a las decisiones de su padre, doña Clara se revela; mientras que doña Inés quiere ser religiosa por su propia voluntad, a doña Clara se lo imponen y no lo acepta; mientras el padre de doña Inés defiende una boda de su hija con don Claudio, el de doña Clara no lo aprueba y aun así se casan, y lo que es más importante, mientras doña Inés refleja la educación tradicional de los hijos, doña Clara es baluarte de los nuevos siglos. Junto con sus padres forman el cuarteto que desarrolla básicamente los tres temas de la obra (el del amor con la ayuda del galán) y, sobre todo, el principal: la educación de los hijos.

A lo largo del estudio de las diferentes partes de la comedia hemos ido apuntando que sus tres temas fundamentales, en orden creciente de importancia, son el amor, las relaciones paterno-filiales y la educación de los hijos. Acabamos de ver, por otro lado, cómo la importancia de los personajes está directamente relacionada con la de los temas.

La vinculación entre los tres temas responde a un eje concéntrico en el que cada tema está jerárquicamente recogido en uno superior, y el superior está dispuesto en función del significado general de la obra. Así, la importancia del amor está puesta al servicio de las relaciones paterno-filiales, que se concretan en la educación de los hijos, tema subordinado al significado general de la comedia. En consecuencia, será necesario explicar la significación de la obra para advertir en orden inverso la intencionalidad de Moratín al componer la comedia y utilizar dichos temas. 
La mojigata de Moratín es una comedia de tesis, pero no se trata de la mera presentación de un tema y de unas conclusiones derivadas, sino que además el tema es aplicable a la contemporaneidad del autor.

El tema de la educación de los hijos estaba siendo fuertemente discutido en la segunda mitad del siglo XVIII, y por eso Moratín, como neoclásico, quiso dejar patente su visión de todo ello. Tradicionalmente la autoridad paterna era inquebrantable, y buena muestra de ello, en lo que a nosotros nos concierne, son las innumerables comedias en las que el padre dice a la hija con quién se tiene que casar, y esta acepta sin más porque ha de dar gusto a su padre y respetar su decisión, aunque no la comparta. Con el neoclasicismo las mujeres comienzan una serie de reivindicaciones que directamente se aplican a la libertad para elegir marido, pues se trata de su propia felicidad. Evidentemente no todos los padres estaban tan dispuestos a aceptar esas nuevas ideas, como es el caso de don Martín, pero la sana rebeldía de las féminas comenzó a forjar, en el último tercio del siglo y primeras décadas del XIX, una libertad de elección desconocida hasta entonces. Y Moratín, neoclásico avanzado, se muestra a favor de esa nueva tendencia, pero es capaz de reflejar en la comedia el extremo opuesto, el de la tradición. Y, quizás destinada tanto a padres como a hijos, propone en su obra que aunque algunos quisieran retrasarlo, la mujer ya tenía derecho a decidir sobre su felicidad.

\section{Conclusiones: Convergencias y divergencias}

Uno de los aspectos en los que más inciden los preceptistas de unas épocas y otras es en los constituyentes estructurales de las obras, donde se encuentra la acción. La obra de Calderón presenta una doble acción (o una acción y su contexto según la terminología que aplica Lope en el Arte nuevo) en la que se demuestra claramente que el género al que pertenece la pieza es la comedia nueva. Tras un lectura atenta de varias comedias observamos perfectamente que no son todas iguales, y una de las diferencias básicas será la reforma calderoniana, tan criticada por los teóricos ilustrados. Calderón propuso una fórmula de enredo en la que las relaciones entre los personajes fueran tan complejas que la solución de los conflictos requiriera una intervención ejemplar o casi divina. La mojigata también recurre a un 
enredo amoroso, pero evidentemente no tiene nada que ver con la complejidad del drama barroco.

Por otro lado, la obra calderoniana responde mucho mejor al esquema aristotélico de planteamiento, nudo y desenlace que la moratiniana, quizás paradójicamente.

Los recursos de composición en La mojigata son cuantitativamente menores que en el drama calderoniano, pero su efectividad mayor. Mientras que Calderón potencia los temas mediante los recursos, Moratín potencia el significado, puesto que los temas están en función del significado en el drama ilustrado. Es de destacar el hecho de que en la pieza barroca predominan los recursos del enredo y los contrastes y en el neoclásico los soliloquios y los comentarios didácticos. Realmente esto responde a estéticas distintas y al mayor o menor propósito moralizador de las comedias.

El número de personajes es similar en ambas obras, y sus funciones también. En las dos hay "galán”, “dama”, “criado”, "gracioso” y “poderoso”, y solamente en la neoclásica encontramos "figurón”. Las funciones están muy claras tanto en Las tres justicias en una como en La mojigata; aunque la relación de los personajes con los temas no es similar. Se ha advertido en el análisis individual que los principales personajes de la comedia de Moratín pueden agruparse por parejas y su importancia es paralela a la de los temas. En la obra de Calderón, en cambio, los personajes están más distantes, sin adecuarse a las normas clásicas de separación de comedia y tragedia (pues aparece un rey y criados). Además, excepto la dama protagonista, el resto de personajes desarrolla básicamente uno solo de los tres temas que aparecen.

Las dos comedias tratan tres temas, y con muchas semejanzas. Las tres justicias en una se encarga del amor, del honor y de las relaciones paternofiliales, mientras que La mojigata analiza el amor, las relaciones paternofiliales y la educación de los hijos. En el fondo, ambos dramas ponen el acento en la educación de los hijos. El hecho de que el del honor no aparezca en la comedia de Moratín puede responder a un gusto de época.

Por otro lado, se advierte que la disposición de los temas no es similar: simétrica en Las tres justicias en una y concéntrica en La mojigata, como ha quedado analizado. Por este motivo el tratamiento de los temas es distinto: el drama barroco se presta más a la individualización de los mismos, mientras que en la pieza ilustrada un tema no puede entenderse sin el otro, 
y ninguno de ellos sin el significado de la obra. El tema fundamental en ambos dramas es la educación de los hijos, aunque se analiza desde una perspectiva distinta: desde el padre en el Barroco, para quien se convierte en una cuestión de honor, y desde la hija en el neoclasicismo, para quien se convierte en una razón vital y ejemplarizante.

En cuanto al significado, relacionado con la educación de los hijos, en ambos casos el padre impone su autoridad y el hijo se rebela. En el Barroco el hijo es condenado a muerte y en la Ilustración la hija se sale con la suya y el padre cede. ¿Cuál es la diferencia? El siglo en el que están escritas.

Estilísticamente, Las tres justicias en una de Pedro Calderón de la Barca y La mojigata de Leandro Fernández de Moratín son totalmente opuestas: la comedia barroca en su máxima expresión y la comedia neoclásica pura. La diferencia entre ambas estéticas es tan grande que se resume en una única palabra: Aristóteles. Los neoclásicos critican fuertemente a Calderón, mucho más que a Lope de Vega, como el corrompedor del género dramático en España. Sin embargo, Calderón impulsó una gran reforma del género y tuvo muchos seguidores.

$\mathrm{Al}$ analizar las poéticas de ambos siglos nos damos cuenta de que Las tres justicias en una es totalmente opuesta a la preceptiva clásica según los neoclásicos, y no lo es tanto según los preceptistas del siglo XVII. Es cierto que obvia conceptos como la división entre comedia y tragedia, la verosimilitud o el decoro, pero respeta, por ejemplo, la unidad de tiempo y, según Lope, también la de acción. La mojigata cumple escrupulosamente los preceptos neoclásicos y tiene reminiscencias barrocas, como el tipo del "figurón". Según la preceptiva barroca el drama de Moratín no hubiera tenido mucho éxito. Sin embargo, se invirtieron los papeles, ¿qué fue lo que ocurrió? El éxito de la comedia, incluso en la época en la que se represente, no depende de su mayor o menor fidelidad a determinadas reglas, sino de la aplicación práctica de su tema principal y su significado.

Las tres justicias en una no tuvo demasiado éxito y Calderón se lamentó toda su vida. Por otro lado, aunque Leandro Fernández de Moratín no tuvo mucho éxito en su vida como dramaturgo, sí lo alcanzó con La mojigata. En los dos casos el tema es el de la educación de los hijos, y en ambos el hijo se rebela al padre, como hemos dicho. La obra del Barroco no triunfa y la del neoclasicismo sí. La razón es muy sencilla: Calderón escribió la suya a mediados del siglo XVII y Moratín estrenó la suya en la primera década del 
siglo XIX. Esa es la diferencia: casi ciento cincuenta años en los que la sociedad española cambió tan tajantemente que opuso el modelo de sociedad tradicional vigente casi desde la Edad Media al que sentó las bases del que tenemos en la actualidad. Hoy, si le proponemos ambas obras a un público sin interés filológico ni teatral, con el único afán de divertirse, el resultado del éxito hubiera sido, sin lugar a dudas, el mismo.

\section{Referencias}

Benabu, I. (1991). On the boards and in the press: Calderón's "Las tres justicias en una". Kassel: Reichenberger.

Calderón de la Barca, P. (1923). Comedias de don Pedro Calderón de la Barca: Colección más completa que todas las anteriores, tomo III. Madrid: Rivadeneyra.

Fernández de Moratín, L. (1825). Obras dramáticas y líricas de D. Leandro Fernández de Moratín, tomo II. París: Augusto Bobée. . (1977). Teatro completo. Madrid: Editora Nacional.

Hilborn, H. W. (1938). A Chronology of the Plays of D. Pedro Calderón de la Barca. Toronto: The University of Toronto Press.

Parker, A. A. (1962). Towards a Definition of Calderonian Tragedy. Bulletin of Hispanic Studies, 39(4), 222-237. 